\title{
Espuma de silicone na prevenção de lesão por pressão por máscara PFF2 (N95) na pandemia de Coronavírus
}

\author{
Silicon foam in the prevention of pressure injuries by the PFF2 (N95) mask in the Coronavirus \\ pandemic
}

Espuma de silicio en la prevención de lesiones por presión por la máscara N95 en la pandemia Coronavirus

\section{Resumo}

Objetivos: Descrever alterações da integridade da pele relacionadas ao uso de máscara com peça filtrante - PFF2, tipo N95 e descrever ação preventiva da espuma de poliuretano revestida de silicone adesivo. Metodologia: estudo observacional qualitativo do tipo relato de casos. Dados coletados em outubro de 2020 em quatro avaliações, com duas participantes enfermeiras, uma negra e uma branca, ambas atuantes na linha de frente da pandemia de COVID19. Resultados: Com a implementação da espuma de alta tecnologia composta de poliuretano e revestida de silicone adesivo nas áreas de pressão exercida pela máscara N95, enquanto medida de prevenção de lesão por pressão, foi possível observar no exame clínico da pele, ausência de eritema nas áreas de proeminência ósseas da face (giba nasal e regiões malares), melhora do microclima da pele (temperatura e umidade), além de ausência de dor e ardência referida quando na ausência da espuma para alívio da pressão. Conclusão: A espuma de poliuretano revestida de silicone adesivo parece controlar os fatores de risco - pressão, fricção, cisalhamento, alteração de microclima, além da dor e ardência, referidas em áreas de contato com máscara. Entretanto, são recomendados estudos clínicos mais robustos e com número de participantes maior para consenso do melhor método preventivo. Reforça-se que o alívio da pressão de 02 em 02 horas ainda é o método mais recomendado na literatura mundial para manter a perfusão e nutrição tecidual.

Palavras-chave: Infecção por Coronavírus; Lesão por pressão; Equipamento de proteção individual; Pandemias; Enfermagem.

\begin{abstract}
Objectives: Describe changes in skin integrity related to the use of a mask with filter part - PFF2, type N95 and describe preventive action of polyurethane foam coated with adhesive silicone. Methodology: qualitative observational study of the type of case reports. Data collected in October 2020 in four evaluations, with two participating nurses, one black and one white, both working on the front line of the COVID-19 pandemic. Results: With the implementation of high-tech foam composed of polyurethane and coated with adhesive silicone in the pressure areas exerted by the N95 mask, as a measure of pressure injury prevention, it was possible to observe in the clinical examination of the skin, the absence of erythema in the areas bony prominence of the face (nasal hump and malar regions), improvement of the skin microclimate (temperature and humidity), in addition to the absence of pain and burning sensation when in the absence of foam to relieve pressure. Conclusion: The polyurethane foam coated with adhesive silicone seems to control the risk factors - pressure, friction, shear, alteration of microclimate, in addition to pain and burning, referred to in areas of contact with the mask. However, more robust clinical studies with a larger number of participants are recommended for consensus on the best preventive method. It is emphasized that pressure relief every 2 hours is still the most recommended method in the world literature to maintain perfusion and tissue nutrition.
\end{abstract}

Keywords: Coronavirus infections; Pressure ulcer; Personal protective equipment; Pandemics; Nursing. 


\begin{abstract}
Resumen
Objetivos: Describir los cambios en la integridad de la piel relacionados con el uso de una mascarilla con parte filtrante - PFF2, tipo N95 y describir la acción preventiva de la espuma de poliuretano recubierta con silicona adhesiva. Metodología: estudio observacional con abordaje cualitativo del tipo reporte de caso. Datos recopilados en octubre de 2020 en cuatro evaluaciones, con dos enfermeras participantes, una negra y una blanca, ambas trabajando en la primera línea de COVID-19. Resultados: Con la implementación de una acción preventiva de lesiones por presión con espuma de poliuretano con silicona adhesiva, se evidenció ausencia de puntos de presión y eritema en las zonas de prominencia ósea resultante del uso de mascarilla N95, mejora del microclima cutáneo y quemazón. Conclusión: La espuma de poliuretano con revestimiento adhesivo de silicona parece disminuir la presión, posiblemente la fricción y el cizallamiento, reduciendo el dolor y el ardor en las áreas de contacto con la máscara. El uso inadecuado o prolongado puede provocar lesiones por presión. Se recomiendan estudios clínicos más robustos para elegir el mejor material para prevenir estas lesiones.
\end{abstract}

Palabras clave: Infecciones por Coronavirus; Úlcera por presión; Equipo de protección personal; Pandemias; Enfermería.

\title{
1. Introdução
}

Em 30 de janeiro de 2020, a Organização Mundial da Saúde (OMS) declarou surto de coronavírus (COVID-19) constituindo emergência de saúde pública internacional. Pouco tempo após, em março de 2020, foi caracterizada como pandemia. Os profissionais de saúde precisaram incluir medidas de precaução padrão para evitar o contágio e a disseminação do vírus (SARS-Cov-2), que ocorre através de gotículas, aerossóis no contato pessoa-pessoa e contatos com mãos, superfícies e objetos contaminados (OPAS, 2020). Consequentemente, foram observados relatos destes profissionais mundialmente sobre aparecimento de lesões por pressão (LP) decorrente da utilização contínua e prolongada de equipamentos de proteção individual (EPI), especialmente em relação ao uso de máscara com peça facial filtrante (PFF2), mas conhecida como N95, trazendo riscos e danos à pele dos profissionais de saúde.

A lesão por pressão é aquela que se localiza em área de dano de pele e tecido mole subjacente à proeminência óssea (NPUAP, 2016). Esse evento adverso pode determinar altos custos no tratamento para o sistema de saúde. Portanto, medidas preventivas devem ser instituídas conforme avaliação de comportamento de risco (BRASIL, 2014). Para isso é fundamental a execução de processos para gerenciamento de riscos, integração de processos de cuidados e cultura de segurança institucional.

A máscara N95 é um respirador particulado com eficácia mínima na filtração de 95\% de partículas transportadas pelo ar de até $0,3 \mu$, utilizada por profissionais de saúde durante a realização de procedimentos indutores de aerossóis, como: intubação, extubação, traqueostomia, ventilação manual, aspiração aberta, reanimação, dentre outros (Tran, Cimon, Severn, Pessoa-Lima, Conly, 2012; WHS, 2020b).

O uso de máscaras é de suma importância para prevenção e controle da infecção e conter o aumento do número de casos, tanto em ambiente doméstico e/ou áreas controladas, quanto em ambientes hospitalares (WHS, 2020a; WHS, 2020b; WHS, 2020c). A proteção dos profissionais de saúde atuantes na linha de frente deve ser uma prioridade, visando assegurar que estes funcionários possam trabalhar prejuízos devido ao uso de equipamentos que se destinam a protegê-los (Moore et al., 2021).

Tendo em vista, que as LP já são conhecidas e estudadas pelos especialistas em pele, houve identificação do problema nesses profissionais. Diante disso, condutas preventivas para controle de riscos eram necessárias, como controle da pressão, fricção, cisalhamento e alteração do microclima da pele, com aumento da temperatura e umidade (NPUAP, 2016). Um estudo multicêntrico realizado acerca de lesões de pele decorrente do uso de máscaras em 161 hospitais na China aponta uma prevalência de 42,8\%, sendo 2,6 lesões por profissional (Jiang et al., 2020).

Lesões decorrentes da utilização deste EPI mostraram-se análogas à lesão por pressão relacionada a dispositivo médico (LPRDM), diferindo do objetivo do dispositivo, que no segundo é aplicado para fins diagnósticos ou terapêuticos. Em ambos os casos, estas lesões estão em conformidade com o padrão ou forma do dispositivo e seu estadiamento, seguindo a 
mesma lógica da lesão por pressão (NPUAP, 2016).

É possível observar manifestações cutâneas em todos os tons de pele, sendo descritas com maior detalhamento em brancos, devido a melhor observação de alterações precoces do tegumento. Este reconhecimento de lesões é mais complexo em pele com maior pigmentação, sendo uma preocupação dos profissionais de saúde na avaliação clínica, pois é sabido que o grau de pigmentação interfere na semiologia dermatológica (Black et al., 2015).

Existem semelhanças nas estruturas básicas e função em todos os tipos de pele, mas também existem variações sutis. Umas dessas variações é a estrutura do estrato córneo, que apesar de terem espessuras semelhantes em ambos os tipos de pele, na pele escura é mais compacto, possui mais camadas celulares, conferindo maior resistência às irritações químicas e caracterizando-se como barreira mais efetiva aos estímulos externos. Este estrato córneo também possui menos lipídeos, cujos níveis se correlacionam inversamente com a perda trans epidérmica de água, fatores que geram diferenças importantes no exame físico da pele. Fica evidente que a pele escura é mais resistente, entretanto existe maior dificuldade em identificar lesões especialmente LP estágio 1 (eritema) (Coleman et al, 2013; Black et al, 2015).

Diante da realidade pandêmica mundial, este estudo foi desenvolvido a fim de contemplar os seguintes objetivos: descrever alterações na integridade da pele relacionadas ao uso do EPI (máscara com peça filtrante PFF2, tipo N95) e descrever ação preventiva da espuma de poliuretano revestida de silicone adesivo.

\section{Metodologia}

Trata-se de estudo observacional de abordagem qualitativa do tipo relato de caso que obteve aprovação no CEP da instituição cuja CAAE 35544020.7.0000.5257 e parecer 4.317.759. As participantes foram duas enfermeiras atuantes na linha de frente do combate ao coronavírus na instituição, sendo uma negra e a outra branca, com faixa etária entre 30-35 anos, ambas sem comorbidades e sem alterações da integridade da pele prévia à intervenção no exame físico. Ambas concordaram em participar mediante leitura e assinatura do termo de consentimento livre e esclarecido (TCLE), conforme resolução 466 de 2012 do Conselho Nacional de Saúde.

Para Ludke e André (2013), a observação nas pesquisas com abordagem qualitativa favorece contato estreito entre o pesquisador e o participante, pois favorece o reconhecimento das experiências e interpretação dos fenômenos estudados.

A coleta de dados ocorreu em um hospital universitário federal no Rio de Janeiro no mês de outubro de 2020. O protocolo de avaliação de pele ocorreu em dois dias consecutivos para cada participante em quatro etapas: avaliação mediante anamnese e exame clínico, para inspeção e palpação pré e pós uso de máscara sem intervenção preventiva no primeiro dia e no segundo dia, avaliação pré e pós aplicação da espuma de poliuretano com silicone adesivo.

Nos dois dias de avaliação da pele das participantes, houve registro fotográfico antes e após o uso da máscara mediante autorização do uso da imagem conforme assinatura do termo de autorização da imagem conforme Resolução $\mathrm{n}^{\circ}$ 466/12 do Conselho Nacional de Saúde.

\section{Resultados e Discussão}

Foram realizados quatro momentos de observação em cada participante, durante suas atividades laborais, em dois dias seguidos. A primeira participante é uma enfermeira branca de 35 anos. No primeiro dia, foi realizada avaliação da pele íntegra por exame físico, analisando-se presença de alterações na integridade da pele antes da aplicação do EPI (máscara N95). Não houve alterações, estando à pele íntegra.

Durante o uso do EPI, foram avaliados os fatores de risco análogos aos relacionados à LPRDM (NPUAP, 2016), podendo ser descritos como: pressão em área da giba nasal pela haste metálica da máscara; pressão e fricção nas áreas de 
contato superior da máscara (áreas zigomáticas); pressão/fricção nas áreas de contatos laterais dos elásticos de fixação da máscara (regiões malares e orelhas) e alteração do microclima da pele sob a máscara devido excesso de umidade gerada na pele pela dificuldade na perspiração dentro da máscara.

Ainda no primeiro dia de observação, houve o segundo exame físico, após 75 minutos utilizando a máscara (Figura 1) e foram identificados todos os fatores de risco referidos anteriormente. Assim, emergiram os seguintes diagnósticos de enfermagem (Herdman \& Kamitsuru, 2018): risco de integridade da pele prejudicada mediante uso da máscara PFF2 (N95) e risco de lesão por pressão - presença de eritema reativo à pressão na giba nasal, confirmado por teste de digito pressão.

Figura 1: Exame físico após 75 minutos de uso da máscara PFF2 sem intervenção preventiva.

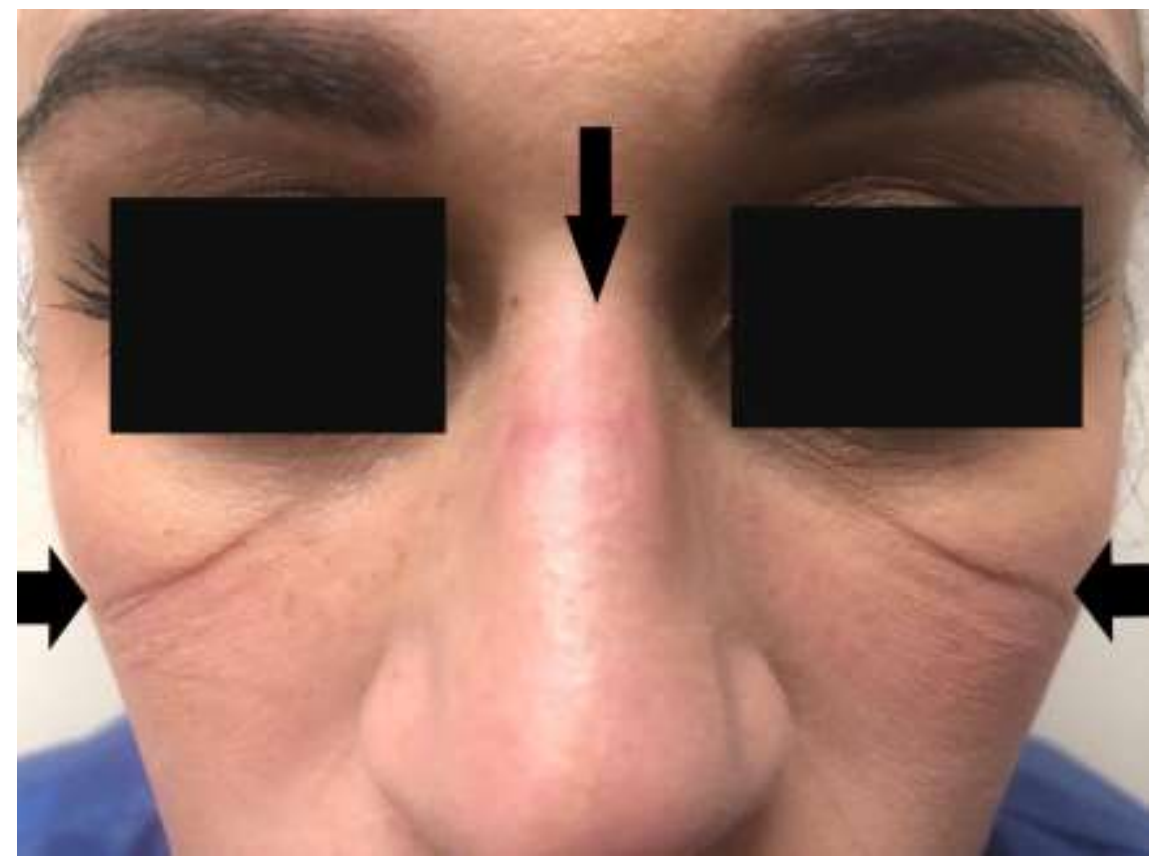

Fonte: Di Piero (2020).

Sobre o teste de digito pressão, vale ressaltar que ele é realizado pelo examinador através da compressão com a polpa digital do dedo indicador por 15 segundos em área de eritema. Se após a retirada do dedo indicador houver branqueamento, resultado negativo. Se eritema não branqueável, diagnostica-se uma lesão por pressão estágio 1 (NPUAP, 2016).

No caso da primeira enfermeira, não houve confirmação diagnóstica de LP secundária ao uso de EPI, provavelmente pelo tempo inferior a duas horas na utilização do EPI tendo em vista período confiável determinado pela literatura científica para prevenção de sofrimento tissular por hipóxia devido excesso de pressão mantida sobre a pele (Brasil, 2014; Black et al, 2015; NPUAP, 2016; WHS, 2020b).

No segundo dia de observação e terceira avaliação da participante, repetiu-se o exame físico da pele para avaliação da integridade e novamente, não apresentou alterações previamente à intervenção preventiva com espuma de poliuretano revestida de silicone adesiva. Foi utilizado o protocolo institucional (Figura 2), orientado pela Comissão de Métodos Relacionados à Integridade da Pele (COMEIP)/Estomaterapia do Hospital Universitário Clementino Fraga Filho da Universidade Federal do Rio de Janeiro (Girondi et al., 2020).

Conforme protocolo, foram realizados três cortes na espuma, sendo maior tamanho para área da giba nasal, passando pelas áreas zigomáticas e outros dois cortes menores para áreas de contato com elásticos da máscara. Após higienização da pele, aplicação e ajustes das espumas adesivas, houve aplicação da máscara PFF2, com realização de teste de pressão 
positiva/negativa (teste de vedação da máscara).

Figura 2: Protocolo de uso da espuma de poliuretano revestido de silicone adesivo.

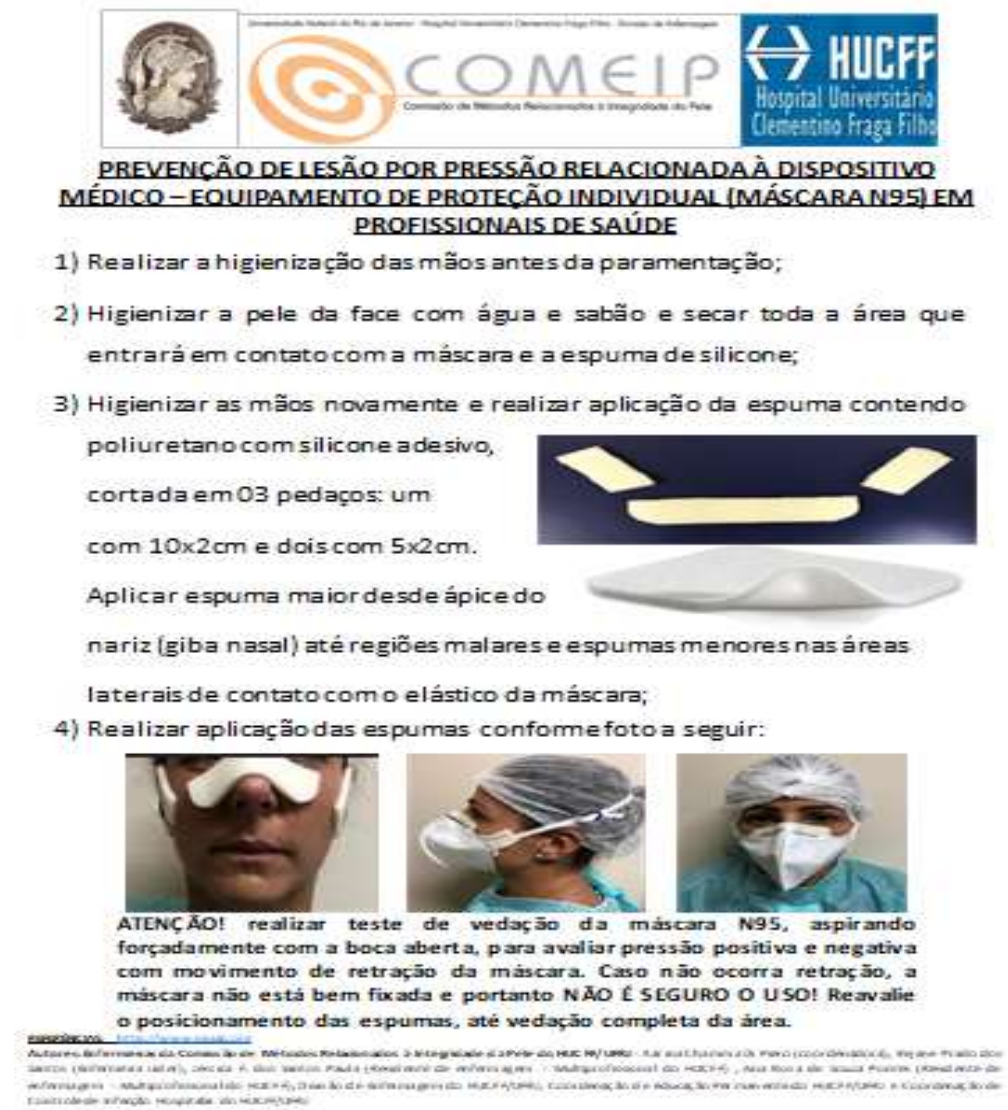

Fonte: Girondi et al. (2020).

A participante permaneceu por três horas utilizando a espuma para prevenção de LP (Figura 3). Após esse período, realizou-se a quarta e última avaliação da pele por exame físico. Não houve eritema reativo ao teste de digito pressão, ou seja, houve redução de áreas de pressão, também visualizada em áreas zigomáticas, com redução das marcas na pele. As áreas de pressão decorrente dos fixadores de elástico laterais não desapareceram, mas foram reduzidas. Também foi constatado melhora da umidade excessiva da pele, referida pela enfermeira e observada pela umidade da espuma, que absorveu o suor retido sob a máscara. Foi referido ainda, ausência de ardência, ou qualquer outro sintoma de desconforto de pressão ou fricção na pele, durante as atividades laborais pela enfermeira. 
Figura 3: Após três horas de uso da máscara PFF2 com espuma de poliuretano revestida de silicone adesivo.

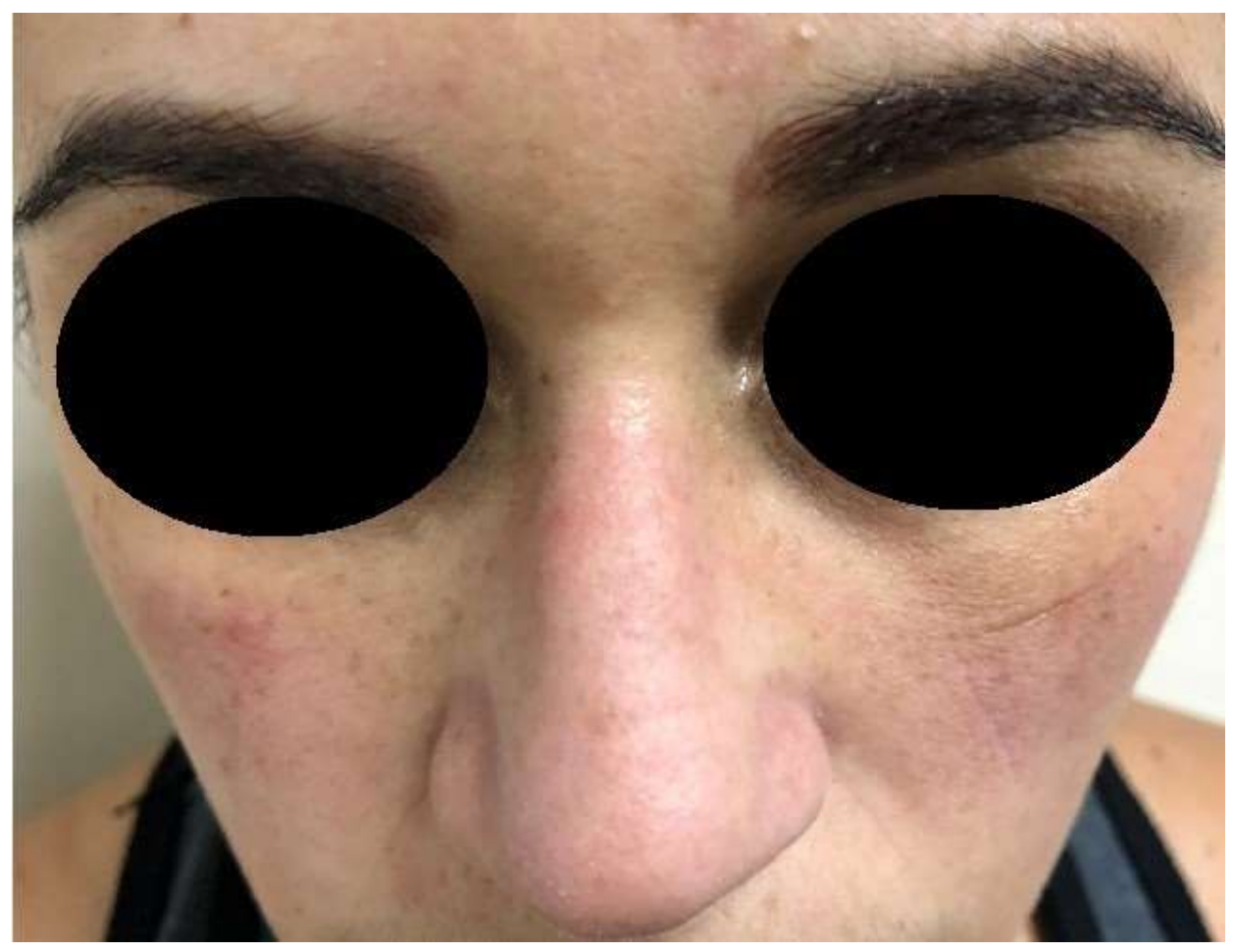

Fonte: Di Piero (2020).

A segunda participante da pesquisa foi uma enfermeira negra de 32 anos que também foi avaliada por exame físico em quatro momentos durante suas atividades laborais por dois dias consecutivos. No primeiro dia, foi realizada uma avaliação da pele através do exame físico visando identificação de alterações na integridade da pele antes da máscara PFF2. Não foram observadas alterações.

Após uma hora utilizando máscara (Figuras 4a e 4b), observou-se no exame físico presença de todos os fatores de risco apontados no relato da primeira enfermeira (pressão, fricção e alteração do microclima da pele). Os mesmos diagnósticos de enfermagem referidos na primeira participante foram realizados na segunda. Embora sendo mais difícil a visualização do eritema reativo a digito pressão presente, este foi pouco visível devido maior resistência da pele escura dada estrutura de seu estrato córneo que confere maior resistência às irritações químicas e barreira mais resistente a estímulos externos (Coleman et al, 2013; Black et al, 2015). 
Figuras 4a e 4b: Exame físico após uma hora de uso da máscara PFF2 sem intervenção preventiva.

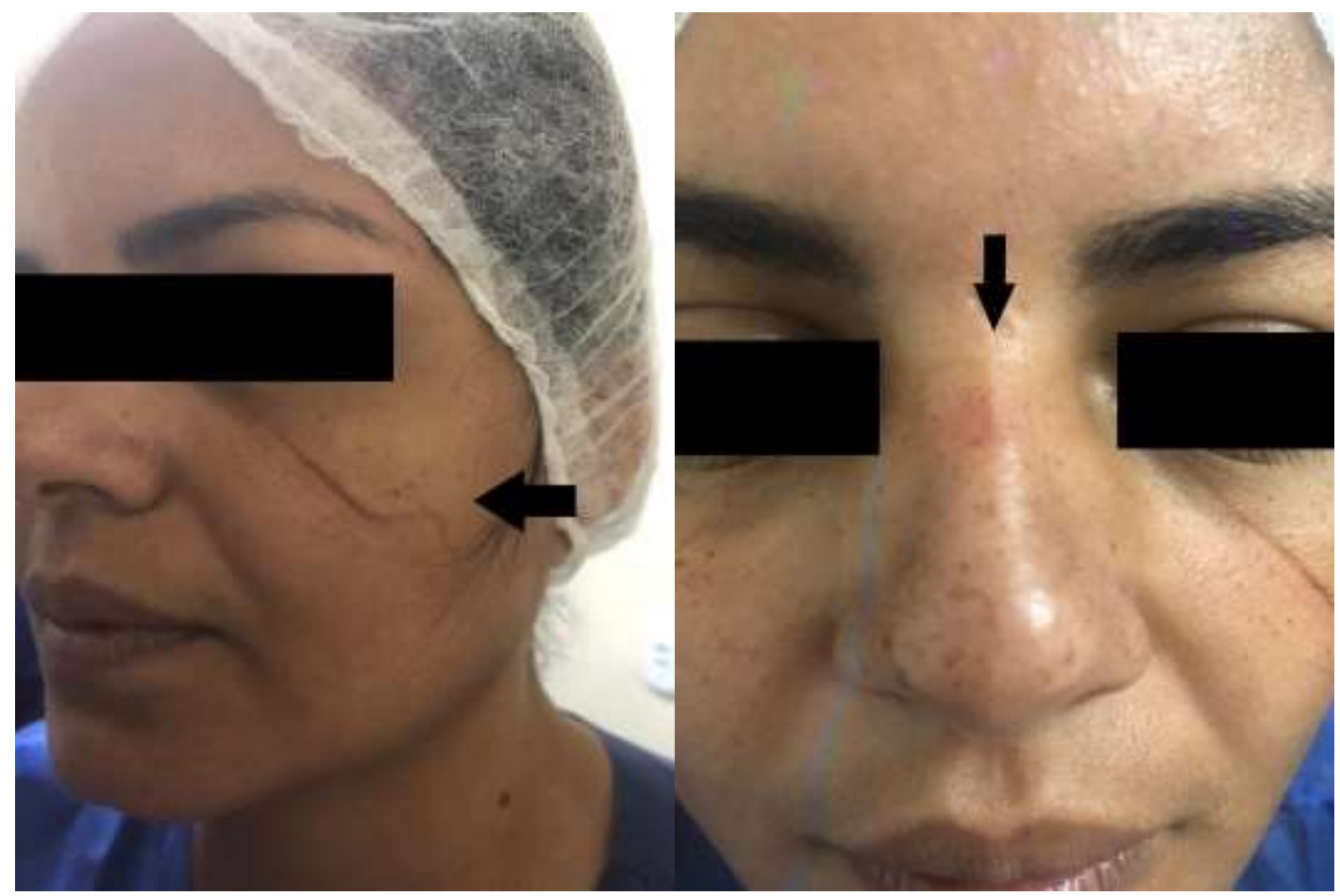

Fonte: Di Piero (2020).

No segundo dia, terceira avaliação, repetiu-se o exame físico da pele e permanecia sem alterações. Assim sendo, foi realizada a intervenção com a espuma de poliuretano revestida de silicone adesivo conforme protocolo. Após duas horas de utilização da espuma sob a máscara PFF2, foi realizada a última avaliação mediante exame físico, que mostrou ausência de eritema (Figura 5), tendo inclusive reduzido áreas de pressão, em regiões zigomáticas, inclusive decorrente dos fixadores de elástico laterais, além da melhora da umidade presente sob a máscara por retenção da perspiração pela espuma e ausência de ardência, ou qualquer outro sintoma de desconforto ao uso do EPI. 
Figura 5: Após duas horas de uso da máscara PFF2 com espuma de poliuretano revestida de silicone adesivo.

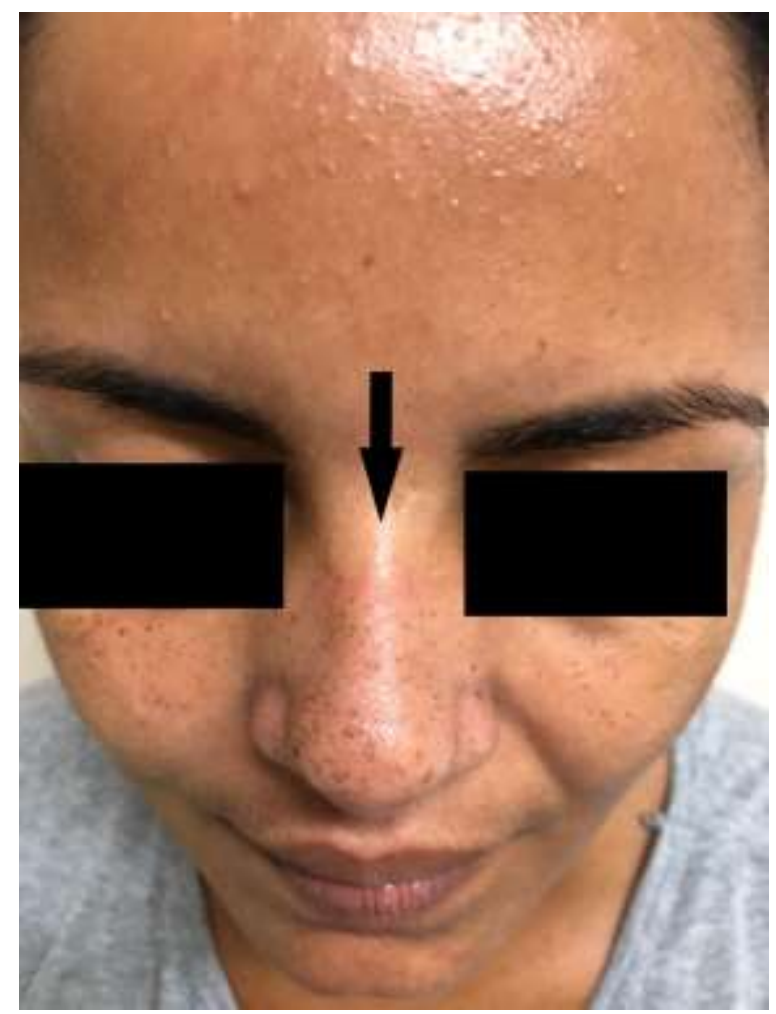

Fonte: Di Piero (2020).

A espuma de poliuretano revestida de silicone adesiva, hipoalérgica e impermeável determinou redução da pressão e fricção, minimizando a umidade presente pela perspiração decorrente de alterações no microclima da pele, com retenção de suor na espuma. Tal situação que evitou hiper hidratação da pele, que poderia evoluir com maceração tecidual e associada ao fator de risco fricção, poderia levar ao desenvolvimento de lesões exulceradas, ou seja, úlceras de pele rasas determinadas pela associação do excesso de umidade e fricção, em decorrência ao uso da máscara PFF2 (N95) (Coleman et al, 2013; Black et al, 2015; NPUAP, 2016; WUWHS, 2016).

Além das espumas, o uso de hidrocolóide extrafino é bastante citado em estudos nacionais e internacionais. Em um estudo realizado com profissionais de saúde do Ceará evidenciou-se o uso de diversos insumos para prevenção de lesões como espumas, filme transparente, hidrocolóide extrafino e fita adesiva/adesivo microporoso, porém, não houve indício comprovação de prevenção de lesões (Coelho et al., 2020).

O relato das enfermeiras sobre a redução expressiva da pressão e ausência da ardência referida previamente ao uso da espuma nas áreas de utilização da tecnologia pode suscitar análise favorável para o equilíbrio das pressões na superfície da pele sob pressão da máscara e fricção, além da umidade já referida.

Outra situação apontada por ambas foi remoção sem trauma na pele das espumas, especialmente sem cisalhar a pele, devido ao revestimento adesivo de silicone. Isto resulta inclusive em redução de dor associada à remoção (Serena et al., 2019). Foi observado que após 24 horas de repouso da espuma em local arejado intra hospitalar, a umidade desaparece da camada de poliuretano do material. Sem gerar impacto negativo na sua forma e função, além de preservar sua camada adesiva de silicone.

Diante disso, suscita-se a discussão de reutilização da tecnologia de alto custo pelo mesmo profissional até a perda da função do material. Entretanto, se houver dúvida sobre contaminação do material, para prevenção de riscos ao profissional, deve-se obrigatoriamente descartar a espuma. É importante salientar que nenhuma das duas participantes contraiu COVID-19 após o uso da intervenção preventiva, mantendo-se a segurança em todas as etapas do estudo. 
Acerca das limitações do estudo, não é possível generalizar e recomendar para todos os indivíduos a mesma tecnologia preventiva. Tendo em vista que o número de participantes pequeno (somente duas participantes) e a suscetibilidade para o desenvolvimento de LP individual. Além disto, o cuidado deve ser individualizado e depende de um exame global com coleta de anamnese, incluindo histórico de hipersensibilidades e alergias e exame físico da pele para identificação de alterações na pele.

Apesar de já existirem evidências internacionais que corroboram com uso de espumas com silicone como insumos preferenciais de prevenção e as considerem categoria de evidência B (EPUAP/NPIAP/PPPIA, 2019; Alves et al., 2020), ainda há controvérsias quanto a generalização na utilização de coberturas de alta tecnologia para prevenção de LP (WUWHS, 2016). A NPIAP (2020) recomenda que não se utilize espumas impermeáveis para que não haja risco de contaminação.

Vale lembrar que as medidas preventivas simples recomendadas com alta força de evidência e recomendação mundial são: descomprimir áreas sob pressão a cada duas horas, hidratar pele com loções cremosas a base de ácidos graxos essenciais (AGE) ou ureia $10 \%$ pelo menos duas vezes por dia, fora do período de utilização da máscara, não massagear áreas com eritema, realizar autoexame da pele diariamente, antes e depois da utilização da máscara PFF2 e realizar teste de digito pressão em áreas de eritema a fim de diagnosticar precocemente LP estágio 1 (NPUAP, 2016).

Por outro lado, nem sempre é possível realizar tais medidas na periodicidade determinada pelos estudos devido à necessidade do profissional de saúde se manter completamente paramentado durante todo atendimento à pacientes com coronavírus e sendo a máscara PFF2 um dispositivo que não pode ser manipulado durante todo o turno. Deste modo devem ser pensadas medidas preventivas, como o uso da espuma em questão, que permanecem por todo o turno de trabalho para alívio da pressão, fricção e umidade.

Foi possível refletir a necessidade de conhecer e controlar os principais fatores de risco relacionados ao desenvolvimento de LP frente ao uso de EPI bem como uso de dispositivos médicos. Diante da impossibilidade de redução do risco com medidas simples referidas nesse estudo, deve-se avaliar a utilização protocolar institucional mediante treinamento de materiais de alta tecnologia, com a reflexão de necessidade individual mediante análise de suscetibilidade e riscos individuais, pois ainda necessitamos de estudos clínicos prospectivos, randomizados e controlados para melhores evidências na prática clínica e generalizações para sua recomendação.

Por fim, o estudo também possibilitou um processo de educação em saúde institucional em relação ao protocolo utilizado na intervenção. Visando prevenir riscos relacionados à LPRDM, houve conscientização da equipe de saúde para o autocuidado por meio do autoexame da pele, orientação do teste de digito pressão em áreas com eritema, especialmente em áreas de risco iminente de lesão e cuidados com contaminação na manipulação do EPI e da espuma.

\section{Considerações Finais}

O uso de espuma de poliuretano com silicone adesivo pode ser útil para reduzir a incidência de lesões por pressão relacionadas ao uso de máscaras PFF2 em profissionais de saúde que estão impossibilitados de realizar medidas simples de prevenção durante suas atividades laborais. Vale lembrar, que a aplicação correta da espuma mediante treinamento é fundamental para o sucesso da prevenção da lesão e para evitar desperdícios do material de alto custo.

Ressalta-se a redução dos fatores de risco (pressão, friçcão, cisalhamento, alteração do microclima, dor e ardência) frente ao uso da espuma sob a máscara N95. Todas as análises são favoráveis para redução de risco, porém há necessidade de estudos de maior magnitude para evidências robustas, tendo em vista seguimento de apenas dois participantes, principal limitação do estudo.

O custo do material, tendo em vista alta tecnologia, pode ser alto para o sistema de saúde. Entretanto se existirem boas 
práticas na sua utilização, pode facilitar padronização do material nas unidades, democratizando seu uso pelos profissionais de saúde.

Conclui-se, portanto, que com este estudo foi possível refletir sobre o tema prevenção de lesões relacionadas à EPI e sua importância para a atuação dos profissionais de saúde que se encontram na linha de frente do combate a COVID-19. Há necessidade de mais estudos, com metodologias mais robustas para comprovação de melhores evidências e recomendações.

\section{Referências}

Alves, P., Gefen, A., Moura, A., Vaz, A., Ferreira, A., Beeckman, D., Malcato, E., Sousa, F., Afonso, G., Kottner, J., Cabete, J., Ramos, P., Dias, V. \& Homem-Silva, P. (2020) PRPPE | COVID 19 - UPDATE. PRevention of skin lesions caused by Personal Protective Equipment (Face masks, respirators, visors and protection glasses). Associação Portuguesa de Tratamento de Feridas. https://dsr.dk/sites/default/files/50/recomendation_prppe_covid19_ing_1.pdf

Bishopp, A., Oakes, A., Antoine-Pitterson, P., Chakraborty, B., Comer, D. \& Mukherjee, R. (2019). The preventative effect of hydrocolloid dressings on nasal bridge pressure ulceration in acute non-invasive ventilation. The Ulster medical journal, $88(1)$, 17. https://www.eadv.org/cmsadmin/showfile/11_Prevention\%20of\%20pressure\%20injuries\%20due\%20to\%20face\%20masks\%20during\%20COVID-19\%20pandemic.pdf

Black, J., Alves, P., Brindle, C. T., Dealey, C., Santamaria, N., Call, E. \& Clark, M. (2015). Use of wound dressings to enhance prevention of pressure ulcers caused by medical devices. International wound journal, 12(3), 322-327. https://doi.org/10.1111/iwj.12111

Brasil. (2014). Documento de referência para o Programa Nacional de Segurança do Paciente/Ministério da Saúde. http://bvsms.saude.gov.br/bvs/publicacoes/documento_referencia_programa_nacional_seguranca.pdf

Coelho, M. D. M. F., Cavalcante, V. M. V., Moraes, J. T., Menezes, L. C. G. D., Figueirêdo, S. V., Branco, M. F. C. C. \& Alexandre, S. G. (2020). Pressure injury related to the use of personal protective equipment in COVID-19 pandemic. Revista Brasileira de Enfermagem, 73 (2), e20200670.

Coleman, S., Gorecki, C., Nelson, E. A., Closs, S. J., Defloor, T., Halfens, R., Farrin, A., Brown, J., Schoonhoven, L. \& Nixon, J. (2013). Patient risk factors for pressure ulcer development: systematic review. International journal of nursing studies, 50(7), 974-1003. https://doi.org/10.1016/j.ijnurstu.2012.11.019

Girondi, J.B.R., Di Piero, K.C., Oliveira, C.F., Rosa, C.S., Santos, L.A. \& Abreu, A.M. (2020). Lesões por pressão relacionada à equipamentos de proteção individual em instituições de longa permanência. In: R. F. Santana (Ed.). Enfermagem Gerontológica no cuidado ao idoso em tempos da COVID-19. 2(1), 126-32 ABEN/DCEG.

Herdman, T.H. \& Kamitsuru, S. (2018). Diagnósticos de Enfermagem da NANDA: definições e classificação 2018-2020/ [NANDA Internacional]. Artmed.

Jiang, Q., Song, S., Zhou, J., Liu, Y., Chen, A., Bai, Y., \& Xue, J. (2020). The prevalence, characteristics, and prevention status of skin injury caused by personal protective equipment among medical staff in fighting COVID-19: a multicenter, cross-sectional study. Advances in wound care, 9(7), $357-364$.

Ludke, M. \& Andre, M. E. D. A. (2013). Pesquisas em educação: uma abordagem qualitativa. São Paulo: E.P.U.

Moore, Z., McEvoy, N. L., Avsar, P., McEvoy, L., Curley, G., O'Connor, T., \& Patton, D. (2021). Facial pressure injuries and the COVID-19 pandemic: skin protection care to enhance staff safety in an acute hospital setting. Journal of wound care, 30(3), 162-170.

National Pressure Injury Advisory Panel. (2020). NPIAP POSITION STATEMENTS ON PREVENTING INJURY WITH N95 MASKS. https://cdn.ymaws.com/npiap.com/resource/resmgr/position_statements/Mask_Position_Paper_FINAL_fo.pdf

National Pressure Ulcer Advisory Panel. (2016). National Pressure Ulcer Advisory Panel (NPUAP) announces a change in terminology from pressure ulcer to pressure injury and updates the stages of pressure injury. https://www.woundsource.com/blog/national-pressure-ulcer-advisory-panel-npuap-announceschange-in-terminology-pressure-ulcer

National Pressure Ulcer Advisory Panel, European Pressure Ulcer Advisory Panel and Pan Pacific Pressure Injury Alliance. (2014). Prevention and Treatment of Pressure Ulcers: Quick Reference Guide. Cambridge Media.

Organização Pan-Americana da Saúde. (2020). Folha Informativa COVID-19 - Escritório da OPAS e da OMS no Brasil. https://www.paho.org/bra/index.php?option=com_content \&view=article \&id=6101: covid19\&Itemid=875

Serena, T. E., Chadwick, P., Davies, P., Johansson, C., Karlsson, C., Edenro, G., \& Sánchez, J. J. S. (2019). Multifunctional and patient-focused Mepilex Border Flex: an exploration of its holistic clinical benefits. Journal of wound care, 28(Sup6b), S1-S31.

Tran, K., Cimon, K., Severn, M., Pessoa-Lima, C. \& Conly, J. (2012). Aerosol generating procedures and risk of transmission of acute respiratory infections to health care workers: a systematic review. PLoS One, 7(4), e35797.

World Health Organization. (2020). Advice on the use of masks in the context of COVID-19: interim guidance, 6 April 2020 (No. WHO/2019nCov/IPC_Masks/2020.3). World Health Organization.

World Health Organization. (2020). Rational use of personal protective equipment for coronavirus disease (COVID-19): interim guidance, 27 February 2020 (No. WHO/2019-nCov/IPCPPE_use/2020.1). World Health Organization.https://apps.who.int/iris/bitstream/handle/10665/331215/WHO-2019-nCovIPCPPE_use-2020.1-eng.pdf

World Health Organization. (2020). Rational use of personal protective equipment (PPE) for coronavirus disease (COVID-19): interim guidance, 19 March 2020 (No. WHO/2019-nCoV/IPC PPE_use/2020.2). World Health Organization. 
Research, Society and Development, v. 10, n. 5, e47510515166, 2021

(CC BY 4.0) | ISSN 2525-3409 | DOI: http://dx.doi.org/10.33448/rsd-v10i5.15166

World Union Wounds Healing Societies. (2016). Documento de Consenso da World Union of Wound Healing Societies (WUWHS). O papel das coberturas na prevenção da lesão por pressão. http://sobende.org.br/pdf/WUHS\%202016/08.pdf 